\title{
Huge cervical fibroid causing urinary retention
}

\author{
Sunita Kumar* \\ Department of Obstetrics and Gynaecology, Sir Ganga Ram City Hospital New Delhi, India
}

Received: 24 August 2016

Accepted: 24 September 2016

*Correspondence:

Dr. Sunita Kumar,

E-mail: drsunitakumar@hotmail.com

Copyright: $\odot$ the author(s), publisher and licensee Medip Academy. This is an open-access article distributed under the terms of the Creative Commons Attribution Non-Commercial License, which permits unrestricted non-commercial use, distribution, and reproduction in any medium, provided the original work is properly cited.

\begin{abstract}
Urinary retention is not so common in females, and when they occur they usually have very predictable reasons depending on the age of the patient and her history. Investigations like CT scan/MRI or videocystourethrography may be needed to come to the exact diagnosis. A pelvic mass filling the pelvis is a relatively common cause, which can be totally cured by surgery. Cervical fibroids form $1-2 \%$ of the total number of fibroids and are usually symptomatic. Symptoms depend on the site and size of the fibroid. Cervical fibroids attaining huge sizes need CT scan/MRI to come to know the exact extent of the fibroid and involvement of the ureter, bladder or rectum. Surgery -myomectomy or hysterectomy in such cases need expertise and experience to avoid damage to these organs.
\end{abstract}

Keywords: Cervical fibroid, Urinary retention

\section{INTRODUCTION}

Urinary retention though not a very common problem in females, could be acute with suprapubic pain or chronic and painless. The different causes of urine retention in a female have predominance according to the age of the patient. Most of the common mechanical causes like a gravid uterus, post-partum, and others like post-operative, UTI and epidural anaesthesia occur at a younger age, as well as causes like pelvic organ prolapse, uterine and cervical fibroids, urethral stenosis, urethral and cervical cancer, anti- incontinence surgery, the neurological causes like detrusor sphincter dysynergia, multiple sclerosis, cerebral palsy, and spinal cord injuries occur at a relatively older age.

A detailed history, physical examination as well as simple investigations like pelvic ultrasound, urinalysis, is usually enough to nail the diagnosis, but certainly the help of cystoscopy, urodynamic studies and an MRI are very useful where the reason is not very obvious. ${ }^{1,2}$

Fibroids in the uterus and cervix are the most common cause for the enlargement of the uterus other than pregnancy. These leiomyomas arise from the smooth muscle of the uterus and the cervix and are usually benign tumours which are oestrogen dependent, hence maximum growth of the tumour is seen during the reproductive age group with the ovarian activity at its peak.

Cervical fibroids form $1-2 \%$ of all the fibroids. They could arise from the anterior, posterior or the lateral wall of the cervix or it could be central involving the whole of the cervix, depending on the size as well as the site of the tumour it may cause symptoms like urinary frequency, urinary retention, constipation, lower abdomen pain, menstrual abnormalities, dyspareunia, and post coital bleeding.

Excision of the cervical fibroid- myomectomy or hysterectomy in patients with cervical fibroid, barring the small sub mucous cervical fibroid are a little difficult to handle due to the proximity of the enlarged cervix to the important structures like the uterine, the ureter, bladder and the rectum. ${ }^{3,4}$

\section{CASE REPORT}

A 53 year old, post-menopausal lady presented with acute retention of urine instead of came to the hospital with a history came to the hospital with a history of acute 
retention of urine. She had a history of repeated urinary retention in the past which was relieved to some extent on its own and occasionally she was catheterized for it. On examination there was a huge mass above the umbilicus. The patient was catheterized and after the bladder was emptied; per vaginal examination was done which revealed an enlarged uterus with mass arising from the cervix, pushing the uterus up and extending laterally into both the fornices and posteriorly. The rectal mucosa was free on per rectum examination.

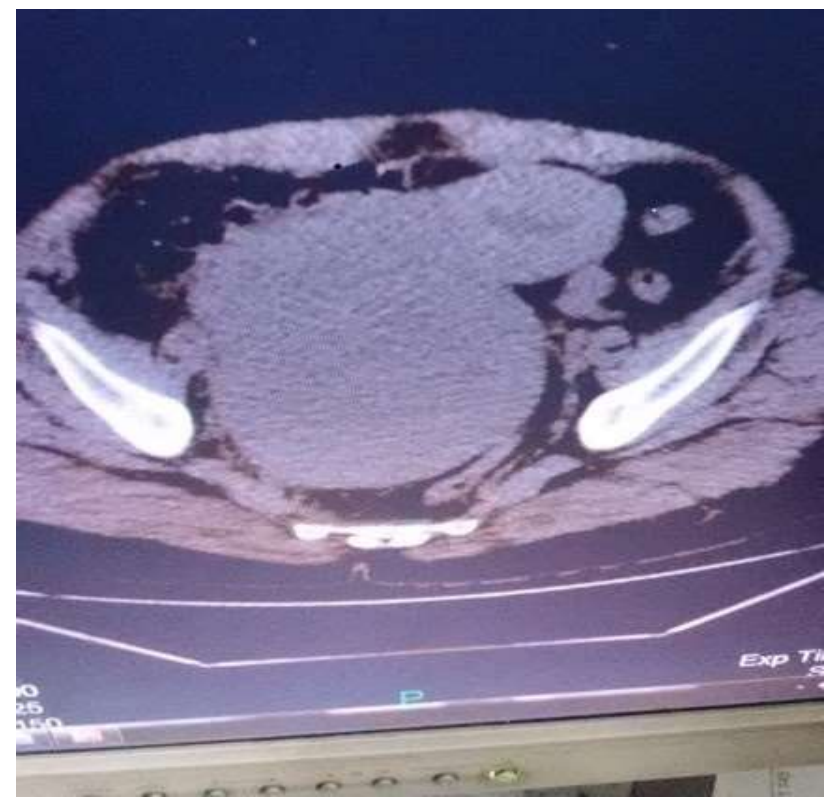

Figure 1: CT scan showing huge cervical fibroid pushing the uterus upwards and to the left.

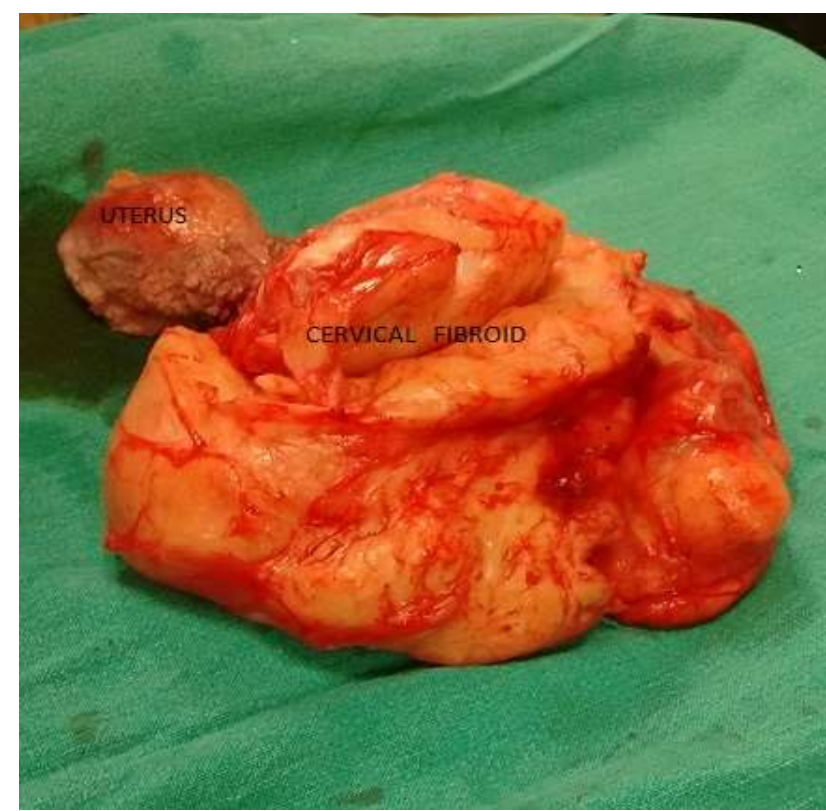

Figure 2: Post-operative specimen showing the uterus and the cervical fibroid.
The ultrasound done showed a huge cervical fibroid, so further evaluation was done with a CT Scan which showed an empty bladder with Foley's bulb in situ and an approximately $12.3 \times 11.2 \times 11.8 \mathrm{cms}$ sized iso-dense, smooth marginated lesion in the pelvis extending from the pelvic floor to almost the bifurcation of the aorta superiorly, with loss of fat planes with the uterus displacing it to the left and displacing the urinary bladder anteriorly. The fat planes between the urinary bladder and the rectum appeared to be maintained (Figure 1).

The patient was taken up for hysterectomy after proper investigations and pre-operative preparations. On opening the abdomen, it was found the uterus was small and a huge cervical fibroid arising from the posterior surface of the cervix and spreading laterally was pushing the uterus upwards. Hysterectomy was routine till the uterine after which the capsule was opened and enucleation of the fibroid attempted, the mass was too big and hence had to be removed after bisecting it transversely. The capsule was adherent to the gut posteriorly which could be removed without much difficulty and once the mass was removed hysterectomy was completed with ease (Figure 2).

Post-operative period was uneventful and the patient was discharged with no urinary symptoms or residual urine.

\section{DISCUSSION}

Small uterine fibroids in postmenopausal female can be left without surgery if there are no symptoms or any changes suggesting of a cancerous growth. But cervical fibroids are usually symptomatic, even the small sub mucous cervical fibroids cause symptoms and need to be removed though they can be tackled vaginaly without much difficulty. Bigger cervical fibroids arising from the anterior or posterior wall of the cervix causing pressure symptoms need special care due to proximity to the various important structures as well as increase in the vascularity. To avoid injury to the important surrounding structures mainly the ureters it's always advisable to dissect the mass intracapsular and proceed with the hysterectomy when the tissues are more or less in the normal anatomical positions. 5

When such masses crowd the pelvic space occasionally the mass fails to clear the promontory of the sacrum and becomes impacted in the pelvis displacing and compressing the lower urinary tract, inciting acute urinary retention. Currently video cystourethrography is the modality for voiding dysfunctions offering both functional and anatomical assessment of the lower urinary tract. Though this is an excellent investigation but comes with a drawback of radiation exposure, cost and not so ready availability. Sonography is reproducible, non-invasive and provides excellent assessment of anatomical changes of the lower urinary tract. ${ }^{6}$ 
This patient had such a huge cervical fibroid with a history of intermittent urinary retention. such patients usually have more problem during the night, as when they are supine the impacted pelvic mass displaces the cervix superiorly and anteriorly compressing the lower bladder leading to obstruction of the internal urethra, during straining the increased abdominal pressure further compresses the lower bladder and though there is no limitation of the urethral mobility the problem worsens, but when such patients stand up, the lower bladder fills with urine and there is descent of the bladder neck relieving the obstruction. ${ }^{7}$

\section{CONCLUSION}

Retention of urine because of mechanical or anatomical reasons can be totally cured by removing the obstruction. The aetiology of huge masses in the pelvis compressing the lower urinary tract is usually difficult and requires the help of CT scan/MRI. Myomectomy or hysterectomy in such cases requires experience and expertise to avoid injury to the important pelvic structures in close vicinity.

Funding: No funding sources Conflict of interest: None declared

Ethical approval: Not required

\section{REFERENCES}

1. Bidzinki M, Siergiel M, Pudkiewiez J, Surynt E, Sikora S. Acute urinary retention due to cervical myoma - a case report and review of the literature. Ginekol Pol. 2015;86(1):77-9.

2. Sethi P, Devi S, Vivekanand A. Acute urinary retention secondary to a cervical leiomyoma -a case report. International Journal of recent trends in Science and technology. 2014;12(1):182-3.

3. Garg R. Two uncommon case presentation of cervical fibroids. People's Journal of scientific research. 2012;5(2).

4. Jayshree V, Mahjabeen M, Imran Mariq. A case of huge cervical fibroid with characteristic lantern on St. Pauls cathedral appearance. Ind Jou of Basi and Appl Med Res. 2015;4(3):455-8.

5. Narmada, Hameed J, Radhika, Geetha, Jaisree. Interesting cases of different types of cervical fibroids. JEMDS. 2014;3(18):4775-80.

6. Jenn-Ming-Yang MD, Wen-Chen Huang, MD. Sonographic findings of acute urinary retention secondary to an impacted pelvic mass. J. Ultrasound Med. 2002;21:1165-9.

7. Eigbefoh J, Onuminyan D, Abebe J. Intermittent urinary retention secondary to a cervical leiomyoma. Trop Jou of Obstet and Gynaecol. 2008;2(2).

Cite this article as: Kumar S. Huge cervical fibroid causing urinary retention. Int J Reprod Contracept Obstet Gynecol 2016;5:4070-2. 